\title{
Grußwort der Psyche
}

\author{
Werner Bohleber
}

Online publiziert: 3. November 2009

(C) Springer-Verlag 2009

Die Psyche gratuliert dem Forum der Psychoanalyse zu seinem 25-jährigen Jubiläum und seiner erfolgreichen Entwicklung zu einem wichtigen Publikationsorgan für die Psychoanalyse. Begonnen hatte das Forum als Zeitschrift für die Psychoanalyse als klinische Theorie und als Grundlagenwissenschaft, was je nach Blickwinkel eine gewisse gegensätzliche oder ergänzende Standortbestimmung zur breiteren konzeptionellen Ausrichtung der Psyche war oder doch so wahrgenommen werden konnte. Denn als Zeitschrift für Psychoanalyse und ihre Anwendungen hatte sich die Psyche schon immer neben der Darstellung des gegenwärtigen Standes der Psychoanalyse, ihrer Forschung, Theoriebildung, Methodologie und Behandlungstechnik auch die Verbreitung von Arbeiten zum Ziel gesetzt, die versuchen, mit psychoanalytischen Mitteln kulturelle, gesellschaftliche und politische Entwicklungen zu durchdringen.

Die Gründungsphase des Forums fällt mit einem Jahrzehnt schmerzhafter und emotional belastender Auseinandersetzungen um die Verstrickung der deutschen Psychoanalytiker in den Nationalsozialismus zusammen. Die Psyche hat bei der „Aufarbeitung“ dieser bis dahin fast gänzlich ausgegrenzten und verdrängten Vergangenheit in den 1980er Jahren eine zentrale Rolle gespielt und dabei nicht nur Lob geerntet: So manchem - auch in der DPV - galt ihre damalige Publikationspolitik als zu ,politisiert“. Doch der Prozess der Selbstaufklärung war nicht mehr aufzuhalten.

In diese Geschichte des allmählichen Aufbrechens überlieferter illusionärer Vorstellungen über die eigene Geschichte in der DPV, dann aber auch in der DPG, scheint ebenso die Idee für das Projekt zu gehören, das zur Gründung des Forums der Psychoanalyse führte. Davon zeugt das von Beginn an bekundete Bestreben, Mitglieder beider Fachgesellschaften zur Zusammenarbeit zusammenzuführen: Dass dies ebenfalls - sicher auch in Abgrenzung zur Psyche - unter der Losung einer zentralen Hinwendung zur Klinik geschah, dürfte seinen Teil zum Erfolg beigetragen haben.

Alles in allem ist die Erfolgsgeschichte des Forums in den letzten zwei Jahrzehnten Zeichen des größeren, umfangreicheren und manchmal nicht einfachen, von mancherlei

Dr. W. Bohleber $(\bowtie)$

Kettenhofweg 62, 60325 Frankfurt am Main, Deutschland

E-Mail: wbohleber@gmx.de 
Vorurteilen und Projektionen behinderten fruchtbaren Verlaufs des Annäherungsprozesses der beiden deutschen psychoanalytischen Fachgesellschaften.

Diese Entwicklung ist auch eine Basis für ein fruchtbares Miteinander der beiden Zeitschriften Psyche und Forum der Psychoanalyse, wobei eine gewisse Rivalität und Konkurrenz durchaus stimulierend sein und helfen können, sich nicht auf erreichten Positionen auszuruhen. Wir wünschen dem Forum in diesem Sinne weiterhin eine erfolgreiche Zukunft.

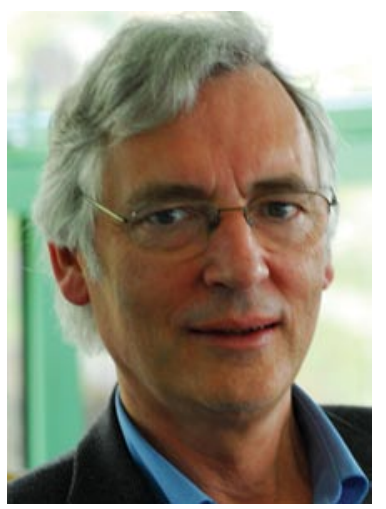

Werner Bohleber, Dr. phil., Psychoanalytiker in eigener Praxis in Frankfurt am Main. Lehranalytiker der Deutschen Psychoanalytischen Vereinigung (DPV), deren Vorsitzender von 2000 bis 2002 . Herausgeber der Zeitschrift Psyche. Veröffentlichungen zur Theorie und Geschichte der Psychoanalyse, zu Adoleszenz und Identität, zur psychoanalytischen Erforschung der nationalsozialistischen Vergangenheit und ihrer Folgen, zu Fremdenhass und Antisemitismus, zur Traumaforschung und zum Terrorismus. 